ARTICLE

\title{
Photoactivatable metabolic warheads enable precise and safe ablation of target cells in vivo
}

\author{
Sam Benson', Fabio de Moliner' ${ }^{1}$, Antonio Fernandez ${ }^{1}$, Erkin Kuru²,3, Nicholas L. Asiimwe (1) 4, Jun-Seok Lee (1) ${ }^{5}$, \\ Lloyd Hamilton ${ }^{6}$, Dirk Sieger (10 ${ }^{6}$, Isabel R. Bravo ${ }^{1}$, Abigail M. Elliot ${ }^{1}$, Yi Feng (ib) ${ }^{1 凶} \&$ Marc Vendrell (i) ${ }^{1 凶}$
}

Photoactivatable molecules enable ablation of malignant cells under the control of light, yet current agents can be ineffective at early stages of disease when target cells are similar to healthy surrounding tissues. In this work, we describe a chemical platform based on aminosubstituted benzoselenadiazoles to build photoactivatable probes that mimic native metabolites as indicators of disease onset and progression. Through a series of synthetic derivatives, we have identified the key chemical groups in the benzoselenadiazole scaffold responsible for its photodynamic activity, and subsequently designed photosensitive metabolic warheads to target cells associated with various diseases, including bacterial infections and cancer. We demonstrate that versatile benzoselenadiazole metabolites can selectively kill pathogenic cells - but not healthy cells - with high precision after exposure to non-toxic visible light, reducing any potential side effects in vivo. This chemical platform provides powerful tools to exploit cellular metabolic signatures for safer therapeutic and surgical approaches.

\footnotetext{
${ }^{1}$ Centre for Inflammation Research, Queen's Medical Research Institute, The University of Edinburgh, Edinburgh, UK. ${ }^{2}$ Department of Genetics, Harvard Medical School, Boston, MA, USA. ${ }^{3}$ Wyss Institute for Biologically Inspired Engineering, Boston, MA, USA. ${ }^{4}$ Molecular Recognition Research Center, Korea Institute of Science and Technology (KIST) \& Bio-Med Program KIST-School UST, Seoul, South Korea. ${ }^{5}$ Department of Pharmacology, Korea University College of Medicine, Seoul, South Korea. ${ }^{6}$ The Centre for Discovery Brain Sciences, The University of Edinburgh, Edinburgh, UK. ${ }^{凶}$ email: yi.feng@ed.ac.uk; marc.vendrell@ed.ac.uk
} 
S ince the early 1990s, photodynamic therapy (PDT) has been clinically used as a therapeutic approach to ablate pathogenic and malignant cells in various diseases, from bacterial infections to cancer ${ }^{1}$. PDT combines light, oxygen, and photosensitizers to produce toxic reactive oxygen species locally, and cause cell death. A broad range of chemical architectures have been reported as effective PDT agents, including organic and inorganic molecules ${ }^{2-6}$, superoxide radical generators ${ }^{7}, 8$, coreshell metal nanoparticles ${ }^{9}, 10$, metal-organic frameworks ${ }^{11-13}$, and mesoporous organosilica nanoparticles ${ }^{14}$. Recently, the groups of Gasser, Chao, and Sadler described the use of highly oxidative iridium photocatalysts to induce intracellular redox imbalance and kill cancer cells even in the presence of low concentrations of oxygen $^{15}$. In addition to exhibiting high phototoxic potential, a critical requirement for PDT agents is to exhibit toxicity only in target cells so that therapeutic benefit can be achieved with tolerable light doses and minimal side effects in healthy tissues.

Different strategies have been described to fine-tune the structures of photosensitizers and enhance their cell selectivity. Activatable agents exploit some of the biochemical features of tumors (e.g., low $\mathrm{pH}$, glutathione, proteases) to release photosensitizers in cancerous tissues, showing promise in reducing bystander toxicity ${ }^{10,16-20}$. Alternatively, photosensitizers can be conjugated to antibodies, which favor accumulation in tissues that express specific cell-surface markers ${ }^{21}, 22$. Whereas these approaches can be effective in the presence of abundant biomarkers, they show limitations at early stages of disease when the biochemical and proteomic signatures of target cells are not dissimilar to those of surrounding healthy tissues (e.g., microtumors). Chemical designs to directly deliver photosensitizers to individual pathogenic and malignant cells under in vivo physiological conditions would significantly extend the clinical applications of PDT.

Cellular metabolism is a reliable indicator of disease onset and frequently used in the clinic (e.g., metabolomics can assist in the detection and therapy stratification of infection ${ }^{23}$ and cancer patients) ${ }^{24,25}$. The metabolic patterns of cells belonging to different taxa (e.g., prokaryotic vs eukaryotic cells) or with altered energetic demands (e.g., cancer vs healthy cells, due to the Warburg effect $)^{26}$ are unique; however, light-controlled ablation of cells on the basis of differential or altered metabolism has been negated by the lack of photosensitive chemical structures that retain the properties of native metabolites.

Most photosensitizers are derived from bulky fluorescent scaffolds ${ }^{27-29}$, which impair their active uptake as photosensitive metabolites. Smaller fluorophores, such as pyrroloindolizines, nitrobenzodiazoles, and naphthylamines ${ }^{30-32}$ are more appropriate to retain metabolite function. In this work, we have designed a platform based on amino-substituted benzoselenadiazoles as the smallest photosensitizers reported to date and used them to build photodynamic agents that recapitulate the uptake of native metabolites, unlike some commercial photosensitizers. Benzoselenadiazole probes can selectively kill cells exhibiting altered metabolism with high spatial resolution and only after exposure to nontoxic light. Importantly, we demonstrate that benzoselenadiazoles are compatible with different metabolite structures (e.g., amino acids, saccharides) and can be used to ablate a variety of disease-causing cells, from bacterial pathogens in vitro to glioblastoma microtumors in vivo. This chemical platform demonstrates how small photosensitizers can harness early metabolic signatures to safely eliminate harmful cells in vivo without damaging healthy tissues.

\section{Results}

Amino-substituted nitrobenzoselenadiazoles are small photosensitizers. In order to generate photosensitizers that would not impair the biomolecular properties of native metabolites, we focused on the nitrobenzodiazole core as an example of a small and uncharged chromophoric scaffold ${ }^{33}$. With the exception of nitrobenzoxadiazoles (e.g., NBD-Cl, Fig. 1a), which are commercially available, we employed the diamine $\mathbf{1}$ to prepare nitrobenzodiazoles including different bridging atoms, namely sulfur, selenium, or carbon (Fig. 1a, full synthetic and characterization details in Supplementary Information). Thio- and selenoderivatives $(3,4)$ were prepared by condensation of the diamine 1 with $\mathrm{N}$-sulfinylaniline or $\mathrm{SeO}_{2}$ respectively, followed by nitration of the position 7 under acidic conditions. The synthetic route for the carbon derivative $\mathbf{5}$ was modified due to the lability of aminoacetals under strong acidic conditions, which would deter the nitration step. Therefore, we treated nitrobenzoselenadiazole $4 \mathrm{~F}$ with $\mathrm{HI}$ and $\mathrm{HCl}$ to remove the selenium atom and performed a $\mathrm{Cu}$-catalyzed condensation with acetone to obtain the corresponding carbon-bridged nitrobenzodiazoles (5). All nitrobenzodiazoles were treated with diethylamine and isolated as the corresponding aminoderivatives (2a-5a, Fig. 1a), which absorb in the visible spectral range. Despite some of these compounds having a relatively high number of $\mathrm{N}$ atoms, all of them fulfill the rule of 3 (i.e., the number of $\mathrm{C}$ and $\mathrm{O}$ atoms divided by the number of $\mathrm{N}$ atoms is equal or larger than 3 ) and therefore are deemed safe to handle and store ${ }^{34}$.

Compounds $\mathbf{2 a}-\mathbf{5 a}$ showed absorbance maxima wavelengths between 480 and $565 \mathrm{~nm}$ (Supplementary Table 1); however, only the selenium derivative (4a) displayed high cellular toxicity after exposure to the light dose generally used in clinical PDT (i.e., 37J $\mathrm{cm}^{-2}$, Fig. $\left.1 \mathrm{~b}\right)^{35}$. Remarkably, the nitrobenzoselenadiazole $4 \mathrm{a}$ displayed minimal cell death in the dark $(<10 \%)$ and almost complete cell ablation ( $>90 \%)$ after irradiation, whereas none of the other compounds (2a, 3a, and 5a) showed significant differences between dark and light-induced cytotoxicity (Fig. 1b). These observations were supported by DFT (Density Functional Theory) calculations (Supplementary Fig. 1). These indicated that, whilst all compounds had sufficient energy to excite ${ }^{3} \mathrm{O}_{2}$ to ${ }^{1} \mathrm{O}_{2}$ via their $\mathrm{S}^{0}-\mathrm{T}^{2}$ transition, the energy levels of the benzoselenadiazole $\mathbf{4 a}$ showed the smallest energy gap for intersystem crossing between $\mathrm{S}^{1}$ and $\mathrm{T}^{2}$ excited states, which favors the occupation of the $\mathrm{T}^{2}$ state and the probability of singlet oxygen production under light irradiation. Furthermore, the degree of spin orbital coupling induced by selenium is 5 -fold higher than for other heteroatoms ${ }^{36}$, which significantly increases the probability of accomplishing this formally forbidden electronic transition. Collectively, these results highlight the heavy selenium atom as a critical feature to endow the nitrobenzodiazole core with unique photosensitive capabilities.

Previous reports have described the importance of the substituents in the position 4 of nitrobenzodiazoles to fine-tune their optical properties ${ }^{37}$. Therefore, we synthesized a subset of nitrobenzoselenadiazoles where the position 4 was functionalized with different nucleophiles and aromatic groups to assess whether those would influence phototoxicity. Benzoselenadiazoles including amines, phenols, thiophenols, and aryl groups (compounds 6-9, Fig. 1c) were synthesized by nucleophilic substitution under basic conditions or by conventional Pd-catalyzed Suzuki couplings (full synthetic and characterization details in Supplementary Information). Spectral characterization confirmed that amino-substituted analogs presented an effective push-pull dipole as indicated by their longer excitation wavelengths (Fig. 1d). Amino-substituted nitrobenzoselenadiazoles showed the highest phototoxicity in cells compared to all other substituents and to the control compound 4F (Fig. 1d). We also examined whether the incorporation of additional heavier atoms in nitrobenzoselenadiazoles-such as iodine, already reported for the successful conversion of other fluorescent structures into photosensitizers ${ }^{38}$-would further 
a

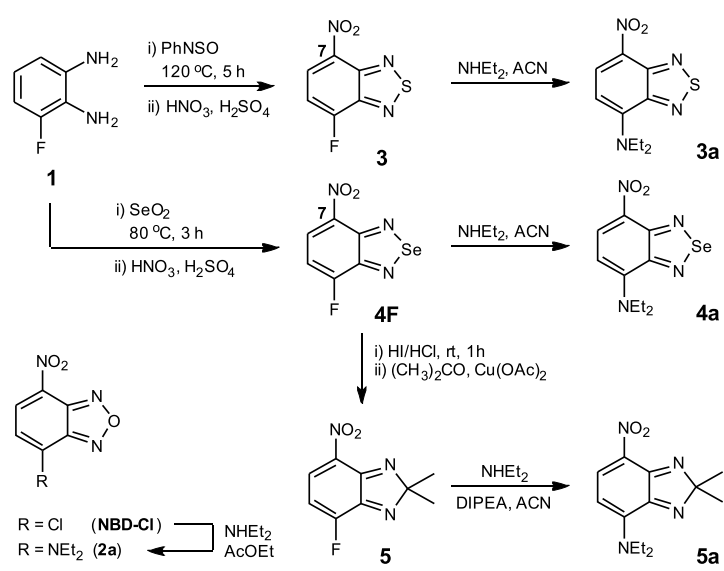

C<smiles>O=[N+]([O-])c1ccc(N2CCCCC2)c2n[n-]nc12</smiles>

6<smiles></smiles><smiles>COc1ccc(Sc2ccc([N+](=O)[O-])c3n[n-]nc23)cc1</smiles>

Substitution at position 4

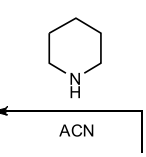

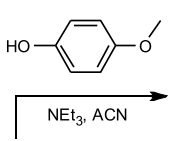<smiles>[R]=[Fe+]</smiles>
$\mathrm{R}=\mathrm{Cl}(4 \mathrm{Cl})$ 4

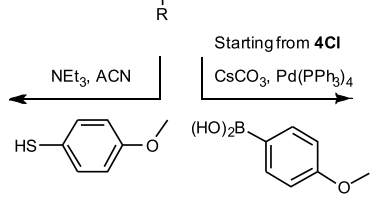<smiles>COc1ccc(-c2ccc([N+](=O)[O-])c3n[n-]nc23)cc1</smiles>

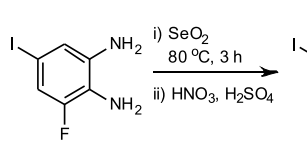

10<smiles></smiles>

11<smiles>COc1ccc(Oc2ccc([N+](=O)[O-])c3n[nH]nc23)cc1</smiles>

d

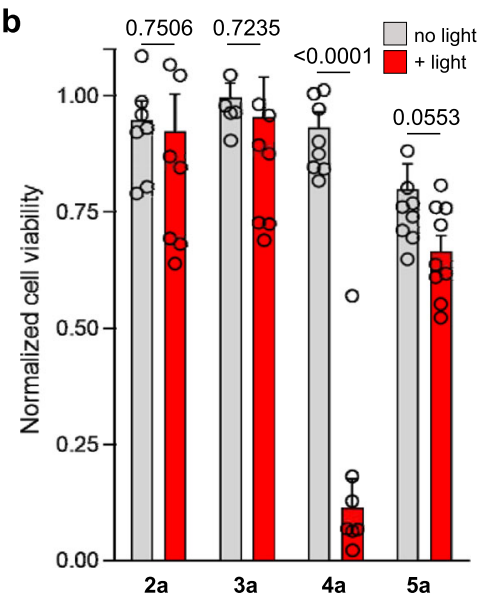

\begin{tabular}{|c|c|c|}
\hline Code & $\lambda_{\text {max. }}$ (nm) & $\begin{array}{c}\text { Photoinduced } \\
\text { toxicity }\end{array}$ \\
\hline $\mathbf{2 a}$ & 490 & $13 \%$ \\
\hline $\mathbf{3 a}$ & 480 & $8 \%$ \\
\hline $\mathbf{4 a}$ & 510 & $82 \%$ \\
\hline $\mathbf{5 a}$ & 565 & $14 \%$ \\
\hline $\mathbf{6}$ & 510 & $64 \%$ \\
\hline $\mathbf{7}$ & 400 & $2 \%$ \\
\hline $\mathbf{8}$ & 440 & $20 \%$ \\
\hline $\mathbf{9}$ & 425 & $27 \%$ \\
\hline $\mathbf{1 1 a}$ & 500 & $65 \%$ \\
\hline $\mathbf{4 F}$ & 442 & $2 \%$ \\
\hline
\end{tabular}

Fig. 1 Synthetic routes for the preparation of small photosensitizers. a Condensation of $\mathbf{1}$ with $\mathrm{PhNSO}$ or $\mathrm{SeO}_{2}$ followed by nitration led to compounds $\mathbf{3}$ and $\mathbf{4 F}$, respectively. Reduction of $\mathbf{4 F}$ followed by Cu-catalyzed addition of acetone rendered compound $\mathbf{5}$. Nucleophilic substitutions of $\mathbf{2 - 5}$ with diethylamine yielded $\mathbf{2 a - 5 a}$. b Human U87 cells were incubated with $\mathbf{2 a - 5 a}(100 \mu \mathrm{M})$ in Krebs-Ringer buffer and illuminated (red bars) or not (gray bars) with a ThorLabs M530L3 LED (10 mW, $37 \mathrm{~J} \mathrm{~cm}^{-2}$, red). Cell viability was assessed $16 \mathrm{~h}$ post illumination using a TACSR MTT cell proliferation assay with values normalized to the viability of untreated cells. Data presented as mean values \pm SEM ( $n=3$ independent experiments). c Nucleophilic substitution of $\mathbf{4 F}$ with piperidine, 4-methoxyphenol, and 4-mehtoxythiophenol led to compounds $\mathbf{6}, \mathbf{7}$, and $\mathbf{8}$, respectively. Suzuki coupling of $\mathbf{4} \mathbf{C l}$ with methoxybenzene4-boronic acid rendered compound $\mathbf{9}$. Condensation of compound $\mathbf{1 0}$ with $\mathrm{SeO}_{2}$ followed by nitration and nucleophilic substitution resulted in compound 11a. $\mathbf{d}$ Maximum excitation wavelengths and phototoxicity of compounds $\mathbf{2 a - 1 1 a}$ and $\mathbf{4 F}$ in human cells as determined in (b). Values indicate the decrease in viability between irradiated and non-irradiated cells after incubation with compounds $\mathbf{2 a - 1 1 a}(100 \mu \mathrm{M})$. $P$ values were obtained from two-tailed unpaired $t$ tests. Source data are available.

improve their phototoxic potential. We employed the commercially available mono-iodinated diamine $\mathbf{1 0}$ to synthesize 9-diethylamino6-iodo-7-nitrobenzoselenadiazole (11a) as described above (Fig. 1c; full synthetic details in Supplementary Information) and compared its behavior to the parent non-iodinated compound (4a). Both compounds 4a and 11a presented similar excitation wavelengths around $500 \mathrm{~nm}$, with the latter exhibiting dark cytotoxicity and the non-iodinated derivative showing higher ratios of phototoxicity, making it more suitable for the preparation of photosensitive metabolites. Finally, we examined the mechanism by which aminosubstituted nitrobenzoselenadiazoles induce oxidative death ${ }^{39}$ and confirmed that, upon light irradiation, they predominantly produce singlet oxygen over radical anions (approximately in a 75:25 ratio,
Supplementary Fig. 2). Amino-substituted nitrobenzoselenadiazoles are therefore one of the smallest photosensitizer structures reported to date.

Benzoselenadiazole-conjugated amino acids retain stereospecific recognition of bacterial cells. In view of the suitable properties of benzoselenadiazoles as small and uncharged photosensitizers, we examined their impact on the molecular recognition properties of metabolites, such as amino acids. VanNieuwenhze, Brun and co-workers have demonstrated that derivatives of D-amino acids (e.g., D-alanine) but not L-amino acids can be recognized by the metabolic cell wall machinery of bacterial cells and incorporated into 


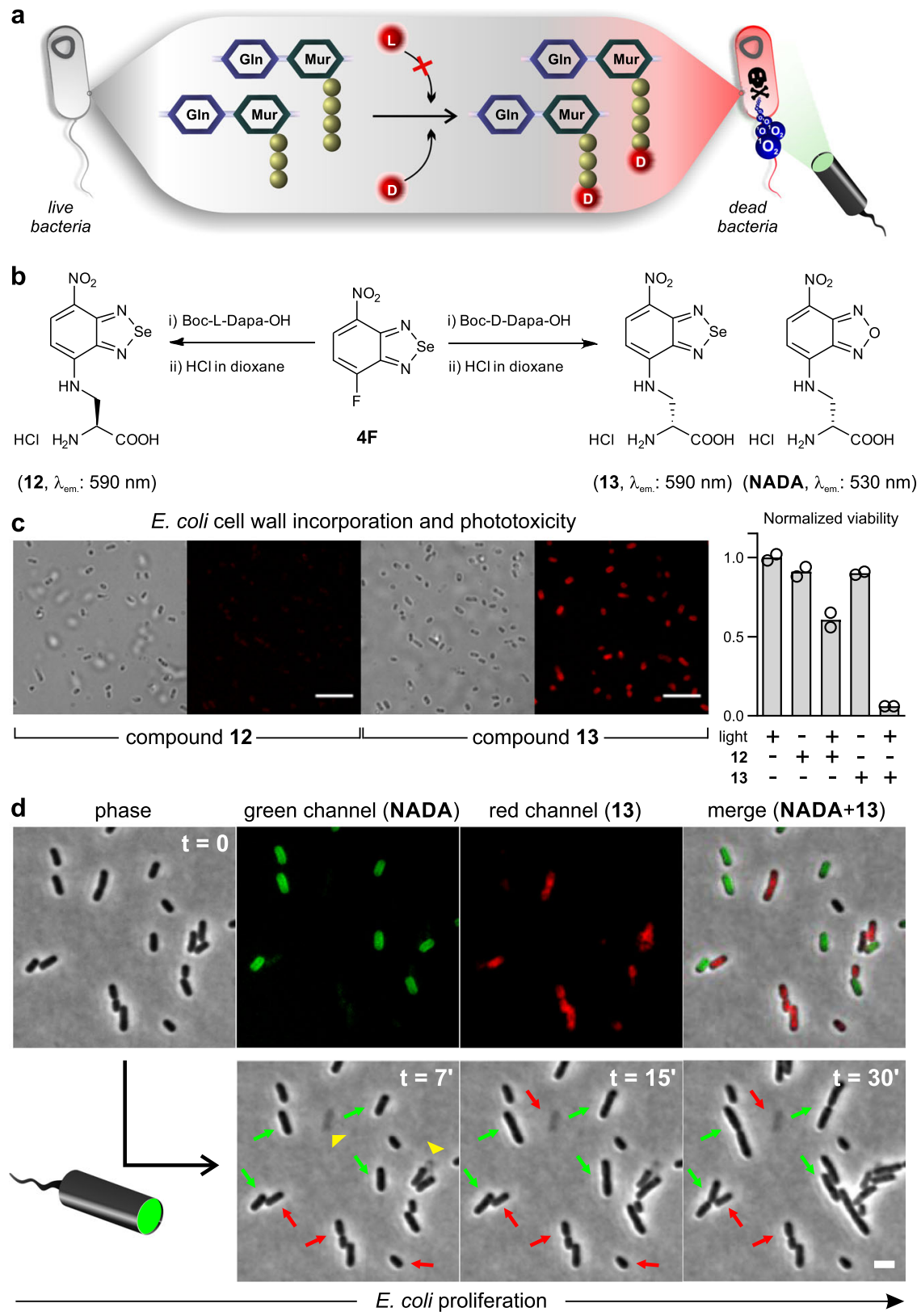

Fig. 2 Benzoselenadiazole D-amino acids can be incorporated into bacteria for light-controlled antimicrobial ablation. a $D$-alanine is incorporated into the positions 4 or 5 of peptidoglycan structures. Incorporation followed by illumination results in the production of singlet oxygen and concomitant cell death (GIn: N-acetylglucosamine, Mur: N-acetylmuramic acid). b Synthesis of the amino acids $\mathbf{1 2}$ and $\mathbf{1 3}$ by conjugation of $\mathbf{4 F}$ with enantiomers of 2,3diaminoproprionic acid. c Brightfield and fluorescence microscopy images of $E$. coli after incubation with compounds $\mathbf{1 2}$ and $\mathbf{1 3}$ ( $250 \mu \mathrm{M}$ ). Scale bar: $10 \mu \mathrm{m}$. The viability of bacterial cells was measured by optical density $(600 \mathrm{~nm})$ after incubation at $30^{\circ} \mathrm{C}$ with or without compounds $\mathbf{1 2}$ or $\mathbf{1 3}$ and illumination using white LED. Data normalized to the viability of light-only treated cells and presented as mean values \pm SEM ( $n=2$ independent experiments). $\mathbf{d}$ Timelapse monitoring of $E$. coli that had been pretreated with NADA or compound 13 (both $100 \mu \mathrm{M}$ ). Representative brightfield and fluorescence microscope images (from three independent experiments) with excitation at $470 \mathrm{~nm}(196 \mathrm{~mW})$ and $508 \mathrm{~nm}(62 \mathrm{~mW})$ were taken every minute for 30 min. Green and red arrows indicate NADA- and 13-labeled bacteria, respectively; yellow arrowheads indicate exploding 13-labeled bacterial cells after treatment. Scale bar: $2 \mu \mathrm{m}$. Source data are available.

their peptidoglycan cell walls (Fig. $2 \mathrm{a})^{40,41}$. Therefore, we prepared benzoselenadiazole derivatives of $\mathrm{D}$ - and $\mathrm{L}$-alanine to examine whether the stereospecific incorporation of the D-enantiomer would be retained after conjugation to the photosensitizer. Two amino acids (12, the L-alanine surrogate and 13, the D-alanine surrogate, Fig. 2b) were synthesized by nucleophilic substitution of the fluorinated benzoselenadiazole $4 \mathrm{~F}$ with the two enantiomers of 2,3-diaminopropionic acid (Dapa) (Fig. 2b, full synthetic details in Supplementary Information). Both amino acids exhibited similar optical properties and singlet oxygen quantum yields, indicating comparable phototoxicity potential (Supplementary Table 1).

During the synthesis of peptidoglycans in the bacterial cell wall, the nascent peptide chains are specifically capped with D-alanines, which can be selectively exchanged by exogenously provided unnatural D-amino acids (Fig. 2a) ${ }^{42}$. To monitor the effect of the amino acids in bacterial cells, we grew Gram-negative Escherichia coli in the presence of compounds $\mathbf{1 2}$ or $\mathbf{1 3}$ and imaged the cells shortly after by fluorescence microscopy. We observed that E. coli 
cells incubated with the D-amino acid 13 -but not the L-amino acid 12- showed uniform labeling of the bacterial septa, indicating that the benzoselenadiazole scaffold retained the selectivity of Dalanine (Fig. 2c). We confirmed by mass spectrometry that the Damino acid 13 was covalently incorporated into the peptidoglycan cell wall (Supplementary Fig. 3) and that the incorporation was independent of the bacterial strain, as shown in experiments with Gram-positive bacteria, such as the model species Bacillus subtillis and the pathogen Staphylococcus aureus (Supplementary Fig. 3). We also examined whether compound 13 could kill E. coli after light irradiation. E. coli bacterial colonies were treated with compounds 12 (as a negative control) or $\mathbf{1 3}$ and kept in the dark or irradiated with light, followed by proliferation measurements under both conditions. As shown in Fig. 2c, high levels of cell death were observed in 13-treated and light-irradiated E. coli cells $(<10 \%$ cell viability) whereas minimal toxicity was detected in non-irradiated cells or cells that had been incubated with the control compound 12. Finally, we performed time-lapse imaging of $E$. coli cocultures that had been separately pre-labeled with compound 13 or NADA (Fig. 2b). NADA is a reported nitrobenzoxadiazole $\mathrm{D}$-alanine analog lacking the selenium atom and able to fluorescently stain bacterial cell walls ${ }^{43}$. The amino acids NADA and 13 fluorescently labeled $E$. coli cells $\left(\lambda_{\mathrm{em}}\right.$ (NADA): $530 \mathrm{~nm}, \lambda_{\mathrm{em}}(13): 590 \mathrm{~nm}$ ), and their growth was monitored for $30 \mathrm{~min}$ while irradiating the cells at regular intervals. Remarkably, most NADA-labeled cells showed clear signs of continued growth and proliferation over time, whereas cells that had been pretreated with compound 13 appeared as lysed or showed arrested cell growth (Fig. 2d). These results support that the local production of singlet oxygen after the irradiation of peptidoglycan-incorporated benzoselenadiazoles can selectively target and kill bacterial cells with high spatial resolution and without affecting neighboring cells in the close vicinity. Given that compound $\mathbf{1 3}$ can be effectively incorporated into Gram-negative and Gram-positive bacteria and cause cell death upon light irradiation, these molecules could open avenues to develop antibiotic regimes overcoming complications associated to antimicrobial resistance in pathogenic bacteria.

\section{A 2-deoxyglucose benzoselenadiazole photosensitizer enables} light-controlled ablation of human glioblastoma cells in vitro and in 3D spheroids. Glioblastomas are the most common and one of the most aggressive types of brain cancer, where surgical treatments require highly precise and safe strategies to remove malignant cells without affecting healthy tissues. Brain tumor cells are very glycolytic and show increased levels of glucose consumption when compared to healthy tissue ${ }^{44}$, hence we decided to assess whether glucose-derivatized benzoselenadiazoles could kill human glioblastoma cells after exposure to nontoxic light.

Two D-glucose analogs (compounds 14 and 15, Fig. 3a) were synthesized by condensation of the benzoselenadiazole scaffold $4 \mathrm{~F}$ with two 2-D-deoxyglucosamine analogs. Both compounds were isolated as the mixture of $\alpha$ - and $\beta$ - anomers of D-glucose (characterization data in Supplementary Information). Compound 15 showed better water solubility and photoreactivity than compound 14, thus we assessed whether it could be actively taken up by GLUT1/3 transporters in glioblastoma cells, the most abundant glucose transporters in human U87 cells ${ }^{45}$. As controls, we also prepared D-glucosamine derivatives of previously reported photosensitizers, such as Nile Blue reported by Fowley and coworkers $^{46}$ and tetraphenylethylene (TPE) reported by the Bin Liu et al. for metabolic $\mathrm{PDT}^{47}$ (compounds 16 and 17, respectively, Fig. 3b). First, we compared the uptake for all D-glucose derivatives in U87 cells. As shown in Fig. 3c, compound $15-$ but not Nile
Blue (16) or TPE (17) derivatives-exhibited reduced uptake in cells that had been pre-incubated with the GLUT inhibitor cytochalasin $B$, suggesting that compound 15-unlike other photosensitizerscan enter glioblastoma cancer cells through active uptake via GLUT transporters. We also analyzed whether the phototoxicity of compound 15 in U87 cells was dependent on GLUT-mediated transport and observed that its activity was significantly blocked with cytochalasin B (Fig. 3c). Furthermore, compound 15 showed the highest singlet oxygen generation quantum yield among all aminobenzoselenadiazoles (Fig. 3d), with values around $25 \%-$ comparable to TPE-and produced effective amounts of singlet oxygen by single-photon $(520 \mathrm{~nm})$ in vitro and in cells as well as by two-photon $(970 \mathrm{~nm}$ ) excitation (Supplementary Fig. 4). Given the technical complexity of two-photon in vivo experiments and the limited availability of multi-photon illumination in clinical settings, we decided to perform our biological characterization using singlephoton excitation.

We cultured human U87 glioblastoma cells and incubated them with compound $\mathbf{1 5}$ to directly image the viability of the cells in the dark as well as after light irradiation. Because photosensitizers producing singlet oxygen induce oxidative cell death via apoptosis $^{48}$, we used the apoptotic marker Annexin $\mathrm{V}$ as an indicator of the toxicity caused by compound 15. Confocal fluorescence microscopy confirmed that U87 glioblastoma cells that had taken up compound $\mathbf{1 5}$ (as shown by intracellular red staining, Fig. 4a) were apoptotic (blue cell membrane staining by Annexin V-Pacific Blue, Fig. 4a) only after light irradiation but not in the dark. On the other hand, single treatments (i.e., compound 15 only or light irradiation only) did not cause cell death as confirmed by fluorescence microscopy and quantitative flow cytometry (Fig. 4b). We also confirmed that compound $\mathbf{1 5}$ would compete with unlabeled D-glucose, which drastically reduced the uptake and phototoxicity of compound 15 (Fig. 4a). Furthermore, as a control we used 2-NBDG, the non-photosensitive nitrobenzoxadiazole glucose analog (Fig. 3a) and confirmed that the selenium atom in the benzoselenadiazole core is essential to cause light-induced oxidative death (Fig. 4b). Finally, we examined the toxicity of compound $\mathbf{1 5}$ in 3D cultures from U87-nlsCrimson glioblastoma cells, which express the fluorescent protein E2Crimson in their nuclei as a cell viability marker $\left(\lambda_{\text {exc }} /\right.$ em: $\left.610 / 645 \mathrm{~nm}\right)$. U87-nlsCrimson cells were cultured in ultra-low adherence plates at $37^{\circ} \mathrm{C}$, forming spheroids of around $250 \mu \mathrm{m}$ in diameter after 3 days. We compared the size of the spheroids in the absence and presence of compound $\mathbf{1 5}$ as well as before and after irradiation (Fig. 4c, d). Compound 15-treated and irradiated spheroids showed a significant decrease in the size of the cell viable core when compared to any of the other incubation conditions, indicating that compound $\mathbf{1 5}$ can effectively kill cells in multiple layers of 3D glioblastoma spheroids.

Compound 15 shows high potency and selectivity for glioblastoma cells in cocultures of human brain cells. In view of the potential of compound $\mathbf{1 5}$ to act as a PDT agent for glioblastoma, we examined its ability to selectively kill glioblastoma cells when they were cultured in the presence of non-cancerous brain cells. We cocultured the glioblastoma cell line U87-nlsCrimson with human $\mathrm{HCN}-2$ cells as representative non-cancerous neuronal precursor cells ${ }^{49}$. HCN-2 cells were pre-stained with CellMask Green and cocultured with U87-nlsCrimson cells (Fig. 5a). Under normal coculture conditions, both $\mathrm{HCN}-2$ and U87 cells were viable, as indicated by fluorescence emission of their respective markers (i.e., CellMask Green for HCN-2, E2Crimson for U87, Fig. 5b). Cocultures were then incubated with compound 15, irradiated with visible light at $37 \mathrm{~J} \mathrm{~cm}^{-2}$, and monitored by fluorescence confocal microscopy. Notably, the combination 
a<smiles>O=[N+]([O-])c1ccc(F)c2n[nH]nc12</smiles>

$4 \mathrm{~F}$
For compound 14: $\mathrm{N}$-ethyl-D-glucosamine $\mathrm{NaHCO}_{3}, \mathrm{ACN}$

For compound 15: D-glucosamine $\mathrm{HCl}$ $\mathrm{NaHCO}_{3}, \mathrm{ACN}$

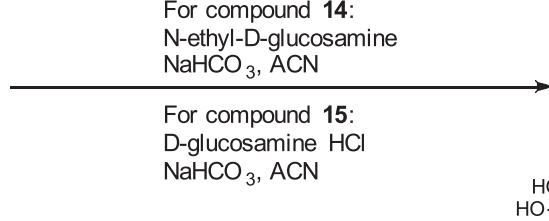<smiles>[R]O[R]([R])=O</smiles>

b

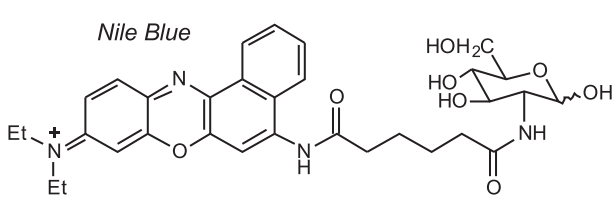

16

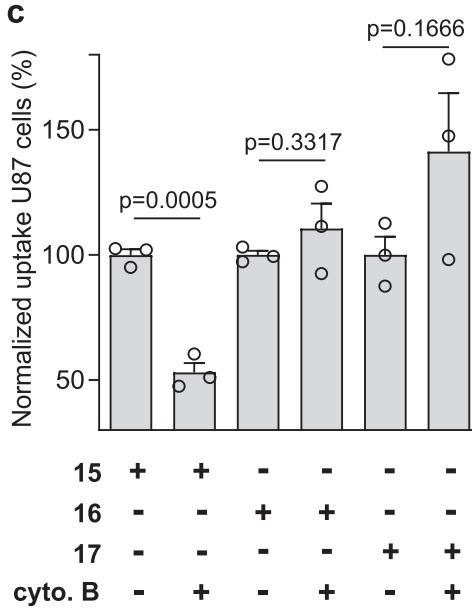<smiles>COc1ccc(C(=C(c2ccc(NC(=O)CCCCC(=O)NC3(C(O)O)OC(C)CC(O)C3O)cc2)c2ccc(OC)cc2)c2ccccc2)cc1</smiles>

d

\begin{tabular}{|c|c|c|}
\hline Compound & $\begin{array}{c}\text { Exc. light } \\
\text { (nm) }\end{array}$ & $\boldsymbol{\Phi}_{\boldsymbol{\Delta}}$ \\
\hline $\mathbf{4 F}$ & 520 & $<1 \%$ \\
\hline 14 & 520 & $4 \%$ \\
\hline 15 & $520,970^{*}$ & $24 \%$ \\
\hline 16 & 640 & $<1 \%$ \\
\hline 17 & white light & $21 \%{ }^{\dagger}$ \\
\hline 5-ALA & 405,640 & $54 \%$ \\
\hline 2-NBDG & 520 & $<1 \%$ \\
\hline
\end{tabular}

Fig. 3 D-Glucose derivatives of benzoselenadiazole - but not of other photosensitizers-are recognized by GLUT transporters. a Chemical synthesis of the D-glucose aminobenzodiazole compounds $\mathbf{1 4}$ and $\mathbf{1 5}$, and the non-photosensitive control compound 2-NBDG. $\mathbf{b}$ Structures of D-glucose derivatives of the photosensitizers Nile Blue (compound 16) and tetraphenylethylene (TPE, compound 17). Full synthetic and characterization details described in the Supplementary Information. c Flow cytometric quantification (gating: Supplementary Fig. 5) of uptake of D-glucose derivatives in human U87 cells (200,000 cells/well) after incubation for $1 \mathrm{~h}$ with compounds 15-17 (50-100 $\mu \mathrm{M})$ in Krebs-Ringer buffer in the presence or not of the GLUT inhibitor cytochalasin B $(20 \mu \mathrm{M})\left(\lambda_{\text {exd }} /\right.$ em $\left.488 / 610 \mathrm{~nm}(\mathbf{1 5}), 635 / 670 \mathrm{~nm}(\mathbf{1 6}), 355 / 450 \mathrm{~nm}(\mathbf{1 7})\right)$. Phototoxicity in U87 cells after incubation with compound 15

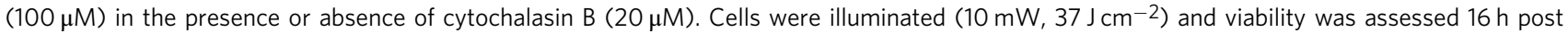
illumination using a TACSR MTT cell proliferation assay with values normalized to those in cells without cytochalasin $B$. Data presented as mean values \pm SEM ( $n=3$ independent experiments). d Excitation wavelengths used for single-photon illumination ("corresponds to two-photon illumination) and singlet oxygen generation quantum yields determined using DPBF in EtOH (Note: DPBF was found insoluble in water) using Rose Bengal as a reference (68\%) 61. $\uparrow$ Singlet oxygen generation quantum yield reported for TPE $62 .{ }^{¥}$ Reported values for protoporphyrin IX, the main photosensitizer produced upon metabolism of 5-aminolevulinic acid (5-ALA) ${ }^{63}$. $P$ values were obtained from two-tailed unpaired $t$ tests. Source data are available.

treatment of compound $\mathbf{1 5}$ and light-induced cell death in most U87 cells-as shown by the lack of fluorescence in the E2Crimson channel-but not in HCN-2 cells, which are less glycolytic and metabolically-active (Fig. 5b). Plot profile analysis confirmed that $\mathrm{HCN}-2$ cells remained intact after the treatment whereas U87 cells were devoid of E2Crimson fluorescence (Fig. 5c). Furthermore, we confirmed that cocultures which had not been treated with compound 15 or not exposed to light showed no signs of death in any cell type (Supplementary Fig. 6). Finally, we analyzed the solubility and phototoxicity profiles of compound 15 and 5aminolevulinic acid (5-ALA, Fig. 5d), the current gold standard in image-guided surgery for brain tumor resection. 5-ALA stimulates the production of protoporphyrin IX in tumor cells and is currently evaluated in clinical trials for postoperative and interstitial PDT ${ }^{50}$. Both compound 15 and 5-ALA showed full water solubility in the high micromolar range, and we did not detect the formation of any aggregates with diameter size $>10 \mathrm{~nm}$. We also compared the in vitro toxicity of different concentrations of compound 15 and 5-ALA in U87 glioblastoma cells and observed that compound $\mathbf{1 5}$ was able to induce significant levels of cell death at similar doses as 5-ALA and with complete lack of toxicity in HCN-2 cells (Fig. 5e). Taken together, these results highlight the biocompatibility and potential utility of compound 15 for light-controlled ablation of cancer cells with comparable phototoxicity to PDT agents under clinical evaluation.

Compound 15 enables safe removal of microtumors in vivo. Encouraged by the selective photoreactivity of compound $\mathbf{1 5}$ in metabolically-active cancer cells, we evaluated whether it could be used to eliminate small tumors derived from human glioblastoma cells in vivo. We used a zebrafish model where low numbers of glioblastoma cells (around $50 \pm 10$ cells per organism) were injected into the yolk sac of 2-day-old embryo and allowed to form microtumors ${ }^{51-53}$. In order to quantify the viability of tumor cells by fluorescence confocal microscopy, we used the above described U87-nlsCrimson cells since they express a live-cell fluorescent reporter that can be detected in the optically-transparent zebrafish. As expected, the injection of U87-nlsCrimson cells into zebrafish 

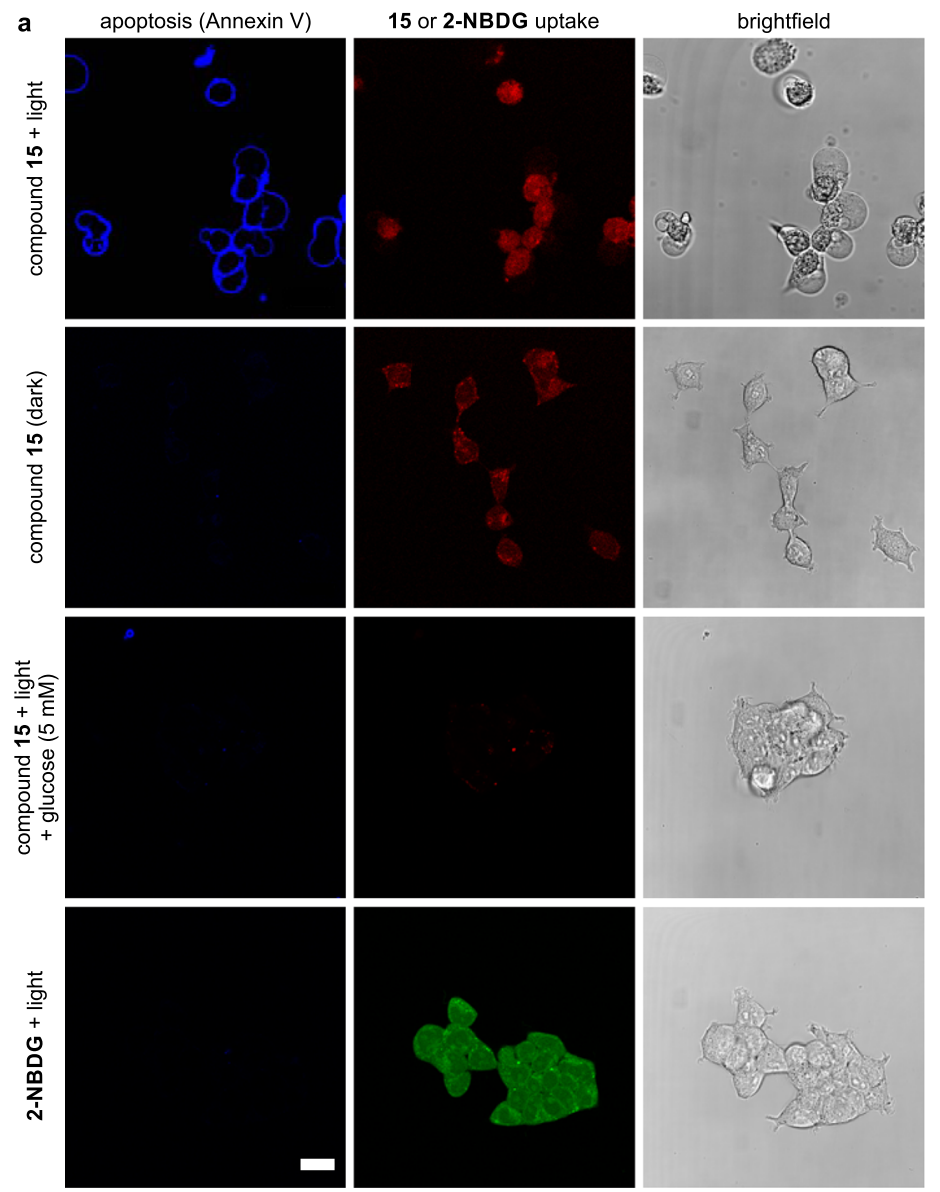
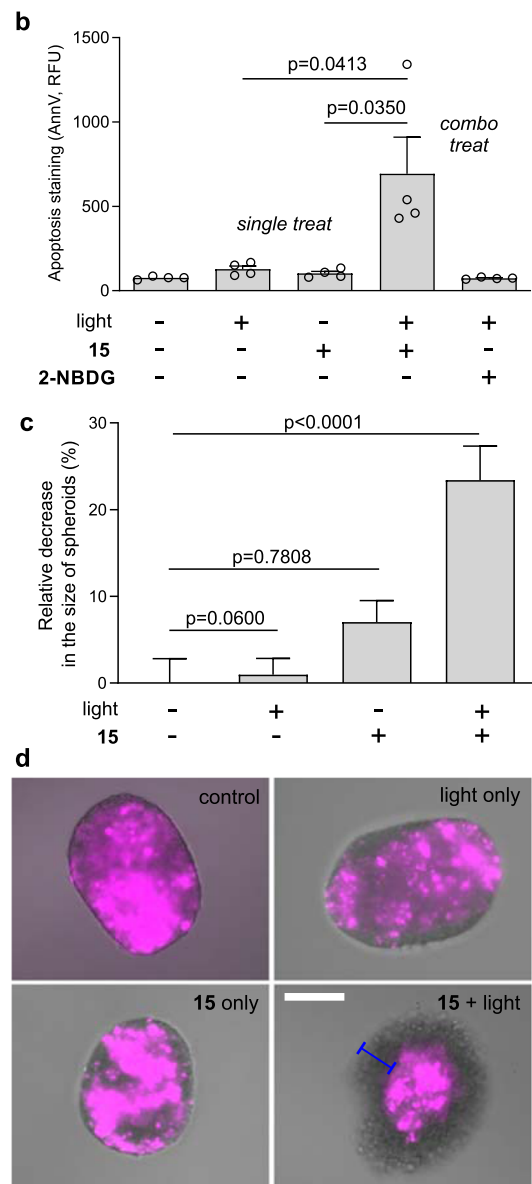

Fig. 4 Compound 15 kills metabolically-active human glioblastoma cells in vitro and in 3D structures. a Representative brightfield and fluorescence images (from three independent experiments) of U87 human glioblastoma cells $\left(20,000\right.$ cells/well) treated with compound $\mathbf{1 5}$ (red, $\lambda_{\text {em }} 590 \mathrm{~nm}$ ) or $\mathbf{2 -}$ NBDG (green, $\lambda_{\mathrm{em}} 530 \mathrm{~nm}$ ) (both $100 \mu \mathrm{M}$ ) with or without D-glucose $(5 \mathrm{mM})$ and illuminated or not with ThorLabs M530L3 LED (10 mW, $37 \mathrm{~J} \mathrm{~cm}^{-2}$ ). Annexin V-Pacific blue $\left(4 \mu \mathrm{g} \mathrm{mL}^{-1}\right.$ ) was used as an apoptosis marker (blue, $\lambda_{\mathrm{em}} 450 \mathrm{~nm}$ ). Scale bar: $10 \mu \mathrm{m}$. b Flow cytometric quantification (gating: Supplementary Fig. 5) of apoptotic U87 cells after different treatments. Data presented as mean values \pm SD ( $n=4$ independent experiments). c Size decrease of U87-nlsCrimson 3D spheroids after variable treatments with compound $\mathbf{1 5}(100 \mu \mathrm{M})$ and visible light $\left(10 \mathrm{~mW}, 37 \mathrm{~J} \mathrm{~cm}{ }^{-2}\right)$. Data presented as mean values \pm SEM and normalized to the viability of untreated spheroids ( $n=3$ independent experiments with three technical replicates).

d Representative merged brightfield and fluorescence microscope images (from three independent experiments) of U87-nlsCrimson spheroids where live cells expressed E2Crimson fluorescent protein ( $\lambda_{\text {em }} 645 \mathrm{~nm}$, magenta). Spheroids treated with compound 15 plus light showed circumference of dead cells of $\sim 50 \mu \mathrm{m}$ in diameter (blue bar). Scale bar: $100 \mu \mathrm{m}$. $P$ values were obtained from two-tailed unpaired $t$ tests. Source data are available.

embryo at $48 \mathrm{hpf}$ (hours post-fertilization) led to the formation of fluorescent tumor mass of several microns in diameter (Fig. 6a). Tumor-bearing zebrafish were then treated with compound $\mathbf{1 5}$ (microinjection of $6 \mathrm{pg}$ into the yolk sac) and illuminated with visible light at a dose of $37 \mathrm{~J} \mathrm{~cm}^{-2}$. Confocal microscopy images were taken from each group, and we quantified the number of remaining fluorescent U87 cells in each larva (Fig. 6a, b). The sequential treatment of compound $\mathbf{1 5}$ followed by light irradiation led to a dramatic reduction of the size of the microtumors, with some zebrafish being entirely free of glioblastoma cells after the treatment (Fig. 6b). The injection of compound 15 or the light irradiation of zebrafish on their own did not cause any significant reduction in the total number of cancer cells (Fig. 6b). Finally, we examined whether the in vivo treatment with compound $\mathbf{1 5}$ caused side effects in zebrafish. First, we compared the morphology of treated and untreated zebrafish, with no evident abnormalities between the two groups (Supplementary Fig. 7). Next, we examined their behavior and vital signs after the PDT treatment. Larvae from control and treated groups showed similar swimming behavior, as shown by the tracks of individual larva ( $n \geq 10$ per group, Fig. $6 c$ ) with no significant differences in distance covered or velocity
(Fig. 6d and Supplementary Movies 1, 2). Heartbeat monitoring in both groups also revealed that treatment with compound 15 and light was safe and did not cause any evident deleterious side effects (Supplementary Movies 3 and 4). In summary, our data indicate that photosensitive metabolites represent a viable chemical strategy to harness the aberrant early metabolic features of aggressive cancer cells and to open avenues for minimally-invasive therapeutic strategies to safely eliminate microtumors.

In summary, this study demonstrates the utility of aminosubstituted benzoselenadiazoles as small photosensitizers to kill metabolically-active cells in vitro and in vivo under full chemical and light control. Unlike in other current photosensitizers, the reduced size of benzoselenadiazoles retains key recognition properties of native metabolites and enables cell-selective uptake via various routes (e.g., peptidoglycan incorporation in bacteria, glucose transport in glioblastoma cells). This chemical approach opens therapeutic avenues for the selective ablation of cells that do not present discriminatory cell-surface markers, such as malignant cancer cells at early stages of disease, or that may become resistant to existing treatments, such as pathogenic bacteria. We also show that the dosing regime and light exposure 

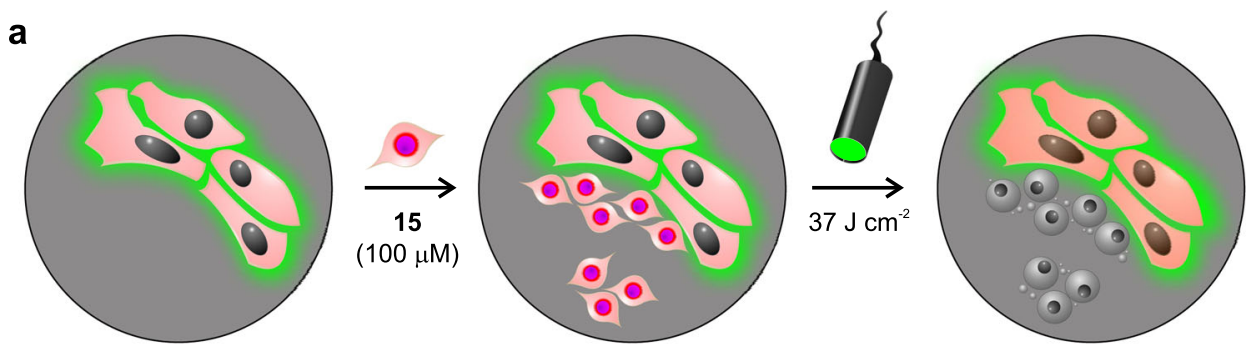

b

co-culture before treatment
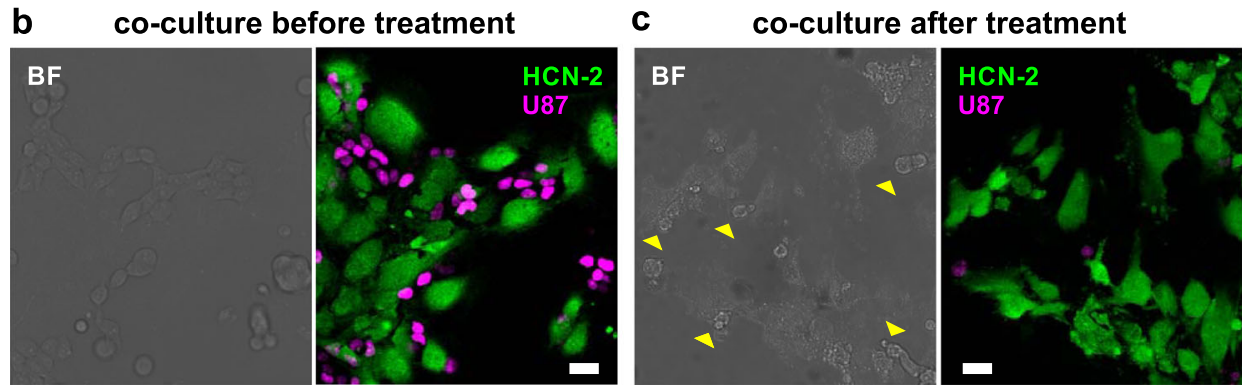

d<smiles>NCCCC(=O)O</smiles>

5-ALA (protoporphyrin IX precursor)

\begin{tabular}{|c|c|c|}
\hline $\begin{array}{c}\text { conc. } \\
(\mu \mathrm{M})\end{array}$ & 5-ALA & 15 \\
\hline 50 & $81 \pm 8 \%$ & $62 \pm 6 \%$ \\
\hline 100 & $92 \pm 2 \%$ & $38 \pm 6 \%$ \\
\hline 200 & $23 \pm 8 \%$ & $16 \pm 5 \%$ \\
\hline
\end{tabular}

e

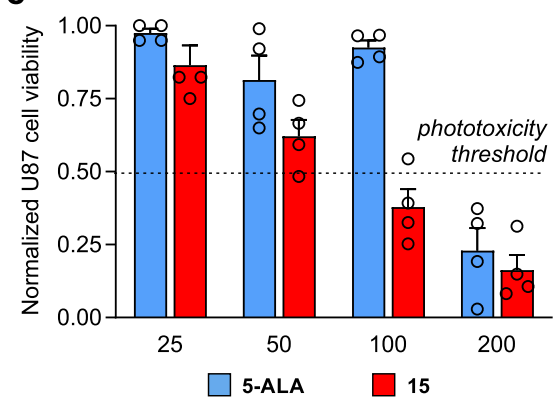

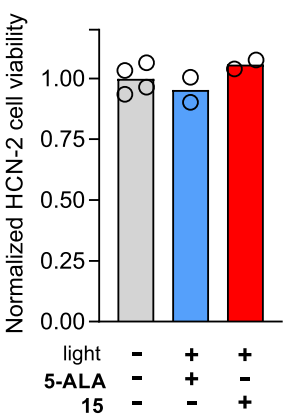

Fig. 5 Compound 15 selectively ablates human glioblastoma cancer cells under clinical PDT conditions and in the presence of non-cancerous human brain cells. a Procedure for coculturing human non-cancerous brain $\mathrm{HCN}-2$ cells (stained with CellMask Green) and human glioblastoma U87-nlsCrimson cells. Cocultures $(15,000 \mathrm{HCN}-2$ cells and 10,000 U87 cells per well) were incubated with compound $\mathbf{1 5}(100 \mu \mathrm{M})$ and illuminated with ThorLabs M530L3 LED $\left(37 \mathrm{~J} \mathrm{~cm}^{-2}\right.$ ). The fluorescence emission from E2Crimson is reduced in U87-nlsCrimson glioblastoma cells upon cell death. (b, c) Representative brightfield and fluorescent confocal microscopy images (from three independent experiments) of cocultured HCN-2 (green, $\lambda_{\text {em }}: 520 \mathrm{~nm}$ ) and U87nls Crimson (magenta, $\lambda_{\text {em }}: 645 \mathrm{~nm}$ ) before (b) and after treatment (c). Yellow arrowheads in (c) highlight dead U87h cells devoid of Crimson fluorescence. Scale bars: $10 \mu \mathrm{m}$. d Phototoxicity analysis under different concentrations of compound $\mathbf{1 5}$ and 5-ALA in U87 glioblastoma cells (20,000 cells/well) using the same irradiation settings $\left(10 \mathrm{~mW}, 37 \mathrm{~J} \mathrm{~cm}^{-2}\right)$. Data presented as mean values $\pm \mathrm{SEM}$ ( $n=3$ independent experiments). e HCN-2 cell viability (10,000 cells/well) after incubation with concentrations under the phototoxicity threshold [i.e., compound $\mathbf{1 5}(100 \mu \mathrm{M}$, red) and 5-ALA (200 $\mu \mathrm{M}$, blue)] and light exposure $\left(37 \mathrm{~J} \mathrm{~cm}^{-2}\right)$. Data presented as mean values \pm SEM $(n=4$ independent experiments). Source data are available.

required for photosensitive metabolites meet high safety standards and are compatible with current procedures used in clinical PDT. The possibility to ablate cells with high precision and purely because of differential or altered metabolism will enable mechanistic studies to identify unknown subsets of diseasecausing cells and to develop safer therapeutic approaches with enhanced spatiotemporal resolution.

\section{Discussion}

The possibility to directly harness aberrant metabolism represents a strategy to improve the selectivity of PDT agents ${ }^{54}$. Photosensitizers that can enter target cells via endogenous metabolic transporters, especially those that are expressed differentially between target cells and their surrounding tissue, would increase selective cell ablation and minimize potential side effects. However, among the many chemical structures reported to date, very few photosensitizers can utilize endogenous metabolic transporters. Herein we describe benzoselenadiazoles as a generic photosensitizer scaffold to produce phototoxic metabolites. The synthesis of aminobenzoselenadiazoles from small metabolites is straightforward, with high-yielding and scalable reactions (Fig. 1). Given that many metabolites contain reactive amino groups (e.g., amino acids, aminoglycans, peptides), this chemical strategy is translatable to a broad range of biological transporters and cell types.

The lack of charged chemical groups and the small size of benzoselenadiazoles are essential to retain recognition and uptake through cell-surface transporters. Aminobenzoselenadiazoles mimic the stereospecificity of chiral amino acids (as shown in L-Ala vs DAla derivatives, Fig. 2) and replicate the uptake of natural biomolecules, as demonstrated with the glucose analog 15 in GLUT transporters (Fig. 3). Of note, GLUT-mediated transport was not observed for other glucose-based photosensitizers, such as Nile Blue or TPE. We have demonstrated that the active uptake of aminobenzoselenadiazoles is essential to 1) minimize off-target toxicity in healthy cells, as shown in human cocultures and in vivo (Figs. 5 and 6), and 2) accumulate concentrations of photosensitizer inside target cells that can generate cytotoxic levels of singlet oxygen under clinical PDT exposure (Figs. 4, 5, and 6). Furthermore, given that aminobenzoselenadiazoles can be excited by single and two-photon 
a
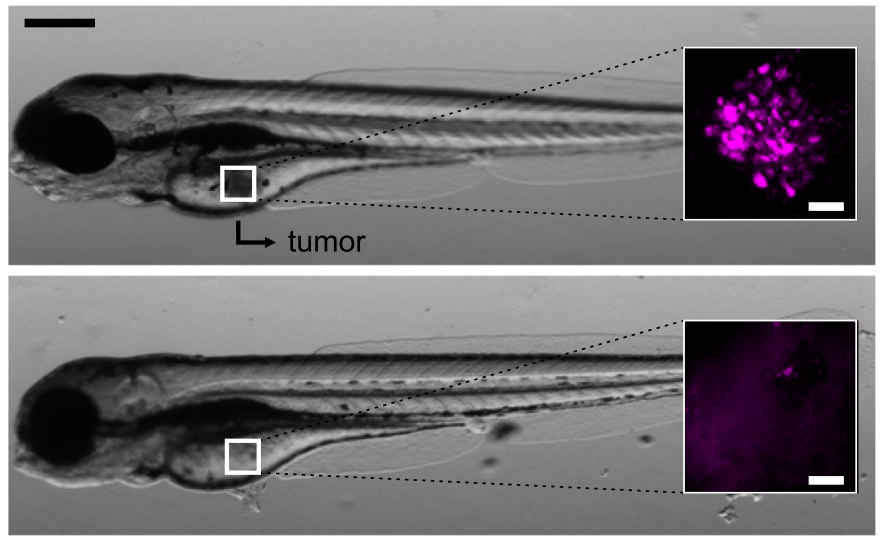

C
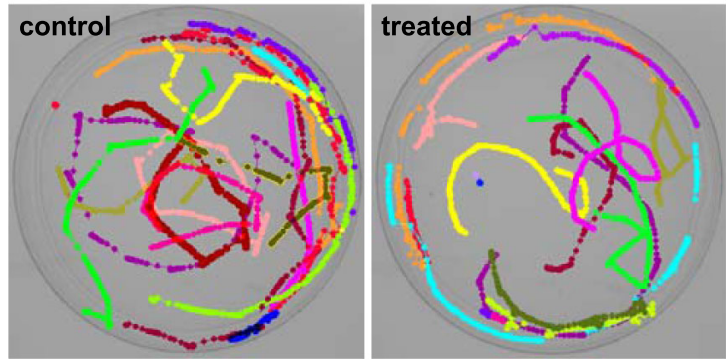

d
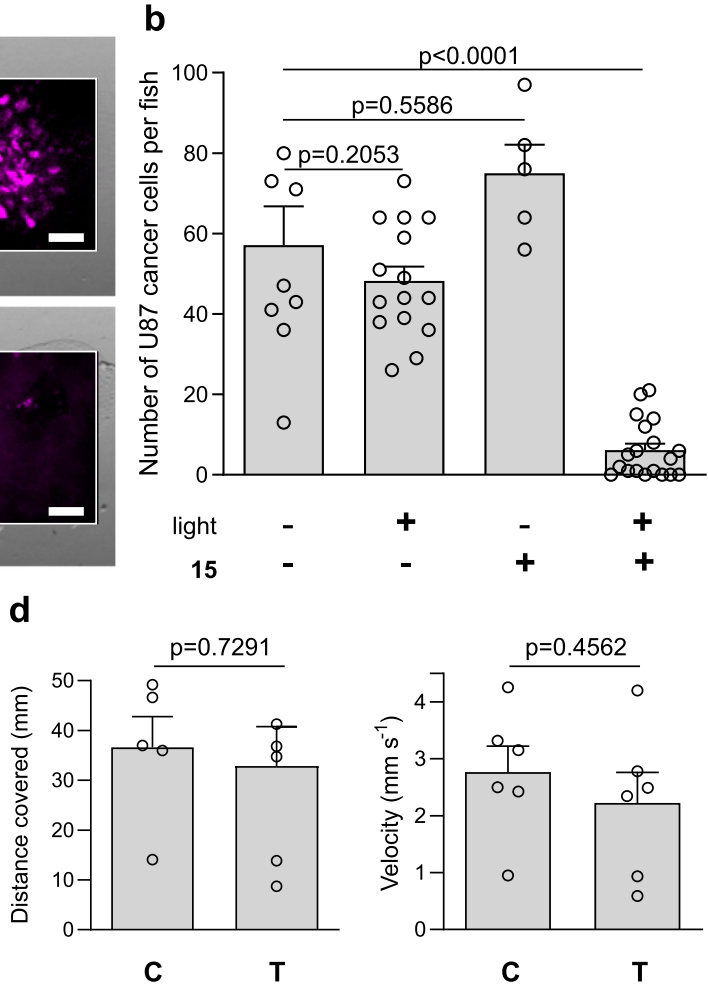

Fig. 6 In vivo treatment of zebrafish xenografts with compound 15 followed by light irradiation leads to safe elimination of microtumors with no evident side effects. a Representative brightfield microscopy images (from five independent experiments) of zebrafish larvae grafted with U87-nlsCrimson

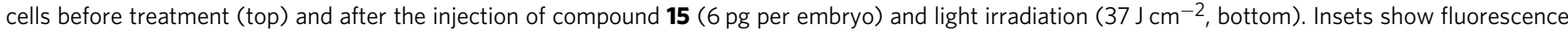
microscopy images $\left(\lambda_{\text {exd }}\right.$ em: $561 / 645 \mathrm{~nm}$ ) of microtumors formed by U87-nlsCrimson cells, which are fully eliminated after treatment. Scale bars: $50 \mu$ m (brightfield), $20 \mu \mathrm{m}$ (fluorescence). b Quantification of fluorescent U87-nlsCrimson cells remaining in zebrafish larvae under different treatments. Data presented as means \pm SEM ( $n=5$ independent experiments). c Microscope images of superimposed pseudo-colored swimming tracks of control untreated zebrafish larvae and double-treated (compound $\mathbf{1 5}$ plus light) larvae ( $n=5$ per group, representative videos for each group shown as Supplementary Movies 1 and 2). d Quantification of distance and velocity of control untreated zebrafish larvae (C) and double-treated zebrafish larvae (i.e., compound $\mathbf{1 5}$ plus light, T) larvae. Data presented as mean values \pm SEM ( $n=5$ independent experiments). $P$ values were obtained from one-way ANOVA with multiple comparisons in (b) and two-tailed unpaired $t$ tests in (d). Source data are available.

sources (Fig. 3 and Supplementary Fig. 4), near-infrared multiphoton endoscopes-increasingly used for in vivo applications ${ }^{55,56}$ - could be considered as excitation sources. Recent in human studies combining local administration of benzodiazoles with endoscope-based imaging suggest that this approach might be translatable to the clinic ${ }^{57}$.

Importantly, aminobenzoselenadiazoles show PDT efficacy at a power of $37 \mathrm{~J} \mathrm{~cm}^{-2}$, which is compatible with clinical use $\mathrm{u}^{58}$. Under these conditions, we have demonstrated that benzoselenadiazole D-amino acids can selectively kill bacterial cells upon irradiation. In mammalian cells, we observed similar cytotoxicity between compound $\mathbf{1 5}$ and 5-ALA, the only FDA-approved photosensitizer able to enter cancer cells through endogenous transporters (i.e., PepT1 and PAT1 transporters) ${ }^{59}$. Unlike 5ALA, compound 15 enters cancer cells within minutes and does not need to be metabolized to be phototoxic (5-ALA is converted to PPIX after several hours). This feature allows local administration of the photosensitizer to minimize systemic side effects, something that cannot be done with 5-ALA. In summary, this chemical platform offers a safe and rapid therapeutic avenue for the metabolic ablation of pathogenic cells, such as early-stage cancers and drug-resistant bacteria, which prove difficult to target with existing PDT approaches.

\section{Methods}

Density Functional Theory (DFT) calculations. B3LYP, PBE0, M06-2X, and wB97XD functionals along with 6-31 G* and def2tzvp basis sets were tested for the closest match between the experimental absorption maxima in ethanol and the energy calculated for the $S^{0} \rightarrow S^{1}$ transitions of compounds 2a-5a. TD-B3LYP/ def2tzvp achieved the closest match and was used for all subsequent computations.

Bacterial cell growth. E. coli, B. subtilis, and S. aureus cells were grown to exponential phase in $\mathrm{LB}$ medium at $37^{\circ} \mathrm{C}$. Cells were incubated with compounds 12,13 , or NADA at the indicated concentrations. Sacculi from labeled cells were isolated by adding cell suspensions to boiling sodium dodecyl sulfate (SDS, $5 \% \mathrm{w} / \mathrm{v}$ ) and incubation for $30 \mathrm{~min}^{41}$. The SDS-insoluble material was collected by ultracentrifugation at $39,000 \mathrm{xg}$ for $10 \mathrm{~min}$ at $30^{\circ} \mathrm{C}$, resuspended in water and boiled again in SDS (4\% w/v) with stirring for $30 \mathrm{~min}$. Samples were then washed, pelleted, and resuspended in the minimal amount of water. Growth curves were measured via absorbance at $600 \mathrm{~nm}$ over $18 \mathrm{~h}$ post-incubation with probes and/or illumination. Time-lapse microscopy experiments were performed with pre-labeled E. coli cells spotted on LB-agarose pads with images acquired every 1 min using a Nikon Ti2 Eclipse inverted microscope equipped with a Lumencor SPECTRA light engine and consecutively irradiating cells under optimized conditions for NADA $\left(\lambda_{\text {exc }} /_{\text {em }}: 470 / 530 \mathrm{~nm}, 196 \mathrm{~mW}\right.$ ) and compound $13\left(\lambda_{\text {exc }} /\right.$ em $\left.: 508 / 590 \mathrm{~nm}, 62 \mathrm{~mW}\right)$.

Cell culture. U87 cells (ATCC), U87-nlsCrimson cells (provided by Dr Dirk Sieger (University of Edinburgh)), and HCN-2 cells (ATCC) were grown in DMEM medium supplemented with $10-20 \% \mathrm{FBS}$, antibiotics (100 $\mathrm{U} \mathrm{mL}^{-1}$ penicillin, $100 \mathrm{mg} \mathrm{mL}^{-1}$ streptomycin), and $2 \mathrm{mM}$ L-glutamine at $37^{\circ} \mathrm{C}$ in a humidified atmosphere with $5 \%$ $\mathrm{CO}_{2}$. Cells were passaged in T-75 cell culture flasks using trypsin-EDTA.

Photoviability experiments. U87 cells were incubated in Krebs-Ringer bicarbonate buffer (KRB) for $2 \mathrm{~h}$, incubated with or without compounds (2a-17) at the indicated concentrations and illuminated or not with either a ThorLabs M530L3/ M625L4 LED or a white light source $\left(10 \mathrm{~mW}, 37 \mathrm{~J} \mathrm{~cm}^{-2}\right)$. Medium was replaced with fresh DMEM, and cell viability was measured after $24 \mathrm{~h}$ using an MTT Cell 
Proliferation assay following the manufacturer's instructions. Values were normalized to the viability of untreated cells and analyzed by GraphPad Prism.

Determination of singlet oxygen quantum yields. Solutions of DPBF $(300 \mu \mathrm{M})$ and compounds $2 \mathbf{a}-\mathbf{1 6}(50 \mu \mathrm{M}$, unless indicated otherwise) in EtOH were illuminated with visible light $(0.3 \mathrm{~mW})$ for $0.5-4 \mathrm{~min}$, followed by absorbance measurements at $410 \mathrm{~nm}$ using a Synergy spectrophotometer. Rates of singlet oxygen production $(k)$ were calculated by plotting change in absorption of DPBF at $410 \mathrm{~nm}$ against time using the equation below.

$$
k=\left(\left(\frac{\ln \left(\mathrm{DPBF} \mathrm{Abs}_{\mathrm{t}}\right)}{\ln \left(\mathrm{DPBF} \mathrm{Abs}_{0}\right)}\right) \ln \left(\mathrm{PSAbs}_{\mathrm{exc}}\right)\right)
$$

Relative singlet oxygen quantum yields were determined using the equation below with Rose Bengal as the reference. $k$ : rates of singlet oxygen production, $\Phi_{\Delta}$ : singlet oxygen quantum yields.

$$
\Phi_{\Delta \mathrm{PS}}=\Phi_{\Delta \mathrm{ref}} \frac{k_{\mathrm{PS}}}{k_{\mathrm{ref}}}
$$

Flow cytometry. U87 cells (300,000 cells/well) were incubated in glucose-free KRB buffer including or not compound $15(100 \mu \mathrm{M})$ for $1 \mathrm{~h}$. Cells were illuminated or not with visible light $(10 \mathrm{~mW})$. Following incubation in fresh DMEM for $16 \mathrm{~h}$, cells were detached, resuspended in KRB buffer, and analyzed by flow cytometry using Annexin V-Pacific Blue ${ }^{\mathrm{TM}}\left(4 \mu \mathrm{g} \mathrm{mL}^{-1}, \lambda_{\text {exc }} / \mathrm{em}: 405 / 450 \mathrm{~nm}\right)$. Flow cytometry data were analyzed using FlowJo.

Live-cell fluorescence confocal microscopy. U87 cells were plated in glass chamber slides (Nunc ${ }^{\mathrm{TM}}$ Lab-Tek $^{\mathrm{TM}}$ II) in supplemented DMEM medium (20,000 cells/well). After $4 \mathrm{~h}$, cells were incubated with or without compounds 15 or 2-NBDG $(100 \mu \mathrm{M})$ in KRB buffer for $1 \mathrm{~h}$ and illuminated or not with visible light $(10 \mathrm{~mW}, 37 \mathrm{~J}$ $\mathrm{cm}^{-2}$ ). Prior to imaging experiments, cells were incubated with Annexin V-Pacific BlueTM $\left(4 \mu \mathrm{g} \mathrm{mL}^{-1}\right)$ and imaged in a Leica SP8 confocal microscope $\left(\lambda_{\text {exc }} / \mathrm{em}\right.$ : $405 /$ $450 \mathrm{~nm}$ for Annexin V, $\lambda_{\text {exd }} / \mathrm{em}: 488 / 530 \mathrm{~nm}$ for 2-NBDG, and $\lambda_{\text {exc }} / \mathrm{em}$ : $488 / 590 \mathrm{~nm}$ for compound 15). All images were processed and analyzed using ImageJ.

Glioblastoma spheroids. U87-nlsCrimson cells were plated in Corning ultra-low attachment 96-well plates (500 cells/well) in supplemented DMEM medium and incubated for $72 \mathrm{~h}$ at $37^{\circ} \mathrm{C}$ in a humidified atmosphere with $5 \% \mathrm{CO} 2$. Spheroids were incubated with or without compound $15(100 \mu \mathrm{M})$ for $1 \mathrm{~h}$ and illuminated or not with visible light $\left(10 \mathrm{~mW}, 37 \mathrm{~J} \mathrm{~cm}^{-2}\right)$ before being washed and imaged on a widefield fluorescence EVOS microscope (Olympus) $\left(\lambda_{\text {exc }} /\right.$ em $: 610 / 645 \mathrm{~nm}$ ). The spheroids diameter was measured after $48 \mathrm{~h}$ incubation at $37^{\circ} \mathrm{C}$ and size decrease measurements were normalized to untreated spheroids. All images were processed and analyzed using ImageJ.

Human brain cell cocultures. HCN-2 cells (15,000 cells/well) were plated in 6-well glass chamber slides (NuncTM Lab-TekTM II) and incubated with CellMask Green for $30 \mathrm{~min}$. U87-nlsCrimson cells $(10,000$ cells/well) were then added to the wells and co-incubated for $4 \mathrm{~h}$ at $37{ }^{\circ} \mathrm{C}$ in a humidified atmosphere with $5 \% \mathrm{CO}$. Cocultures in glucose-free KRB buffer were incubated with or without compound 15 for $1 \mathrm{~h}$ and illuminated or not with visible light, followed by imaging in Leica SP8 confocal microscope $\left(\lambda_{\text {exc }} / \mathrm{em}\right.$ : $488 / 520 \mathrm{~nm}$ for CellMask Green and $\lambda_{\text {exc }} /_{\text {em }}$ : 561/645 nm for E2Crimson). All images were processed and analyzed using ImageJ.

In vivo zebrafish experiments. Experiments were carried out in accordance with Committee-approved ethical procedures. $48 \mathrm{hpf}$ larvae were anesthetized using MS222 and mounted in 3\% methylcellulose. U87-nlsCrimson ( $\sim 50$ cells/fish) were grafted into the edge of the yolk sac of larvae using a glass needle. Successfullygrafted larvae were selected under a dissecting fluorescence microscope. Larvae were injected with compound 15 (6 pg/embryo) and illuminated or not with visible light $\left(10 \mathrm{~mW}, 37 \mathrm{~J} \mathrm{~cm}^{-2}\right)$. The tumor mass in grafted larvae was imaged at $24 \mathrm{~h}$ posttreatment by embedding in low-melting agarose and acquiring fluorescence images of U87-nlsCrimson cells in a Leica SP8 confocal microscope $\left(\lambda_{\text {exc }}\right.$ em: $561 /$ $645 \mathrm{~nm}$ ). Images were processed and analyzed using Imaris. Zebrafish were handled and maintained in the BVS aquatic facility at the University of Edinburgh according to standard protocols ${ }^{60}$. Animal experimentation was approved by the ethical review committee of the University of Edinburgh and the Home Office in accordance with the Scientific Procedure Act 1986.

Reporting summary. Further information on research design is available in the Nature Research Reporting Summary linked to this article.

\section{Data availability}

The source data underlying Figs. 1b, 2c, 3c, 4b, c, 5e, 6b, d and Supplementary Figs. 2 and 4 are provided as a Source data file. Additional data that support findings of this study are available from the corresponding authors upon reasonable request. Source data are provided with this paper.
Received: 24 October 2020; Accepted: 19 March 2021; Published online: 22 April 2021

\section{References}

1. Wainwright, M. et al. Photoantimicrobials - are we afraid of the light? Lancet Infect. Dis. 17, 49-55 (2017).

2. Matera, C. et al. Photoswitchable antimetabolite for targeted photoactivated chemotherapy. J. Am. Chem. Soc. 140, 15764-15773 (2018).

3. Durantini, A., Greene, L. E., Lincoln, R., Martínez, S. R. \& Cosa, G. Reactive oxygen species mediated activation of a dormant singlet oxygen photosensitizer: from autocatalytic singlet oxygen amplification to chemicontrolled photodynamic therapy. J. Am. Chem. Soc. 138, 1215-1225 (2016).

4. Zhou, J. et al. Highly emissive self-assembled BODIPY-platinum supramolecular triangles. J. Am. Chem. Soc. 140, 7730-7736 (2018).

5. Nguyen, V. N. et al. An emerging molecular design approach to heavy-atomfree photosensitizers for enhanced photodynamic therapy under hypoxia. $J$. Am. Chem. Soc. 141, 16243-16248 (2019).

6. Gorman, A. et al. In vitro demonstration of the heavy-atom effect for photodynamic therapy. J. Am. Chem. Soc. 126, 10619-10631 (2004).

7. Li, M. et al. Near-infrared light-initiated molecular superoxide radical generator: rejuvenating photodynamic therapy against hypoxic tumors. J. Am. Chem. Soc. 140, 14851-14859 (2018).

8. Li, M. et al. Superoxide radical photogenerator with amplification effect: surmounting the Achilles' heels of photodynamic oncotherapy. J. Am. Chem. Soc. 141, 2695-2702 (2019).

9. Duan, X. et al. Photodynamic therapy mediated by nontoxic core-shell nanoparticles synergizes with immune checkpoint blockade to elicit antitumor immunity and antimetastatic effect on breast cancer. J. Am. Chem. Soc. 138, 16686-16695 (2016)

10. Wang, P. et al. Engineered cell-assisted photoactive nanoparticle delivery for image-guided synergistic photodynamic/photothermal therapy of cancer. ACS Appl. Mater. Interfaces 11, 13935-13944 (2019).

11. Mao, D. et al. Metal-organic-framework-assisted in vivo bacterial metabolic labeling and precise antibacterial therapy. Adv. Mater. 30, 1706831-1706838 (2018).

12. Lan, G. et al. Nanoscale metal-organic framework overcomes hypoxia for photodynamic therapy primed cancer immunotherapy. J. Am. Chem. Soc. 140, 5670-5673 (2018)

13. Lan, G. et al. Titanium-based nanoscale metal-organic framework for type I photodynamic therapy. J. Am. Chem. Soc. 141, 4204-4208 (2019).

14. Yang, Z. et al. Precision cancer theranostic platform by in situ polymerization in perylene diimide-hybridized hollow mesoporous organosilica nanoparticles. J. Am. Chem. Soc. 141, 14687-14698 (2019).

15. Huang, H. et al. Targeted photoredox catalysis in cancer cells. Nat. Chem. 11, 1041-1048 (2019)

16. Chiba, M. et al. An activatable photosensitizer targeted to $\gamma$ glutamyltranspeptidase. Angew. Chem. Int. Ed. 56, 10418-10422 (2017).

17. Li, X. et al. A tumor-pH-responsive supramolecular photosensitizer for activatable photodynamic therapy with minimal in vivo skin phototoxicity. Theranostics 7, 2746-2756 (2017).

18. Turan, I. S., Cakmak, F. P., Yildirim, D. C., Cetin-Atalay, R. \& Akkaya, E. U. Near-IR absorbing BODIPY derivatives as glutathione-activated photosensitizers for selective photodynamic action. Chem. Eur. J. 20, 16088-16092 (2014).

19. Tong, H. et al. Glutathione activatable photosensitizer-conjugated pseudopolyrotaxane nanocarriers for photodynamic theranostics. Small 12, $1-10$ (2016)

20. Piao, W. et al. Development of an azo-based photosensitizer activated under mild hypoxia for photodynamic therapy. J. Am. Chem. Soc. 139, 13713-13719 (2017).

21. Stuchinskaya, T., Moreno, M., Cook, M. J., Edwards, D. R. \& Rusell, D. A. Targeted photodynamic therapy of breast cancer cells using antibodyphthalocyanine-gold nanoparticle conjugates. Photochem. Photobiol. Sci. 10, 822-831 (2011).

22. Fernandes, S. R. G., Ferandes, R., Sarmento, B., Pereira, P. M. R. \& Tomé, J. P. C. Photoimmunoconjugates: novel synthetic strategies to target and treat cancer by photodynamic therapy. Org. Biomol. Chem. 17, 2579-2593 (2019).

23. Antti, H. et al. Metabolic profiling for detection of staphylococcus aureus infection and antibiotic resistance. PLoS ONE 8, e56971 (2013).

24. Larkin, J. R. et al. Early diagnosis of brain metastases using a biofluidsmetabolomics approach in mice. Theranostics 6, 2161-2169 (2016).

25. Yuan, B. et al. A plasma metabolite panel as biomarkers for early primary breast cancer detection. Int. J. Cancer 144, 2833-2842 (2018). 
26. Vander Heiden, M. G., Cantley, L. C. \& Thompson, C. B. Understanding the Warburg effect: the metabolic requirements of cell proliferation. Science 324, 1029-1033 (2009).

27. Kamkaew, A. et al. BODIPY dyes in photodynamic therapy. Chem. Soc. Rev. 42, 77-88 (2013)

28. Lu, K., He, C. \& Lin, W. Nanoscale metal-organic framework for highly effective photodynamic therapy of resistant head and neck cancer. J. Am. Chem. Soc. 136, 16712-16715 (2014).

29. Gao, Y. H. et al. Synthesis and evaluation of novel chlorophyll a derivatives as potent photosensitizers for photodynamic therapy. Eur. J. Med. Chem. 187, 111959 (2020).

30. Kim, E., Koh, M., Ryu, J. \& Park, S. B. Combinatorial discovery of full-colortunable emissive fluorescent probes using a single core skeleton, 1,2dihydropyrrolo[3,4-]indolizin-3-one. J. Am. Chem. Soc. 130, 12206-12207 (2008).

31. Benson, S. et al. SCOTfluors: small, conjugatable, orthogonal and tunable fluorophores for in vivo imaging of cell metabolism. Angew. Chem. Int. Ed. 58, 6911-6915 (2019).

32. Su, L. et al. Cu(I)-catalyzed 6-endo-dig cyclization of terminal alkynes, 2bromoaryl ketones, and amides toward 1-naphthylamines: applications and photophysical properties. J. Am. Chem. Soc. 141, 2535-2544 (2019).

33. Uchiyama, S. et al. Fluorescence on-off switching mechanism of benzofurazans. Org. Biomol. Chem. 1, 1067-1072 (2003).

34. Bräse, S., Gil, C., Knepper, K. \& Zimmermann, V. Organic azides: an exploding diversity of a unique class of compounds. Angew. Chem. Int. Ed. 44, 5188-5240 (2005).

35. Vignion-Dewalle, A. S. et al. Red light photodynamic therapy for actinic keratosis using $37 \mathrm{~J} / \mathrm{cm} 2$ : Fractionated irradiation with $12.3 \mathrm{~mW} / \mathrm{cm} 2$ after 30 minutes incubation time compared to standard continuous irradiation with $75 \mathrm{~mW} / \mathrm{cm} 2$ after 3 hours incubation time using a mathematical modeling. Lasers Surg. Med. 49, 686-697 (2017).

36. Herman, F., Kuglin, C. D., Cuff, K. F. \& Kortum, R. L. Relativistic corrections to the band structure of tetrahedrally bonded semiconductors. Phys. Rev. Lett. 11, 541-545 (1963)

37. Thooft, A. M., Cassaidy, K. \& Vanveller, B. A small push-pull fluorophore for turn-on fluorescence. J. Org. Chem. 82, 8842-8847 (2017).

38. Yogo, T., Urano, Y., Ishitsuka, Y., Maniwa, F. \& Nagano, T. Highly efficient and photostable photosensitizer based on BODIPY chromophore. J. Am. Chem. Soc. 127, 12162-12163 (2005).

39. Dolmans, D., Fukamura, D. \& Jain, R. Photodynamic therapy for cancer. Nat. Rev. Cancer 3, 380-387 (2003)

40. Hsu, Y. P. et al. Fluorogenic D-amino acids enable real-time monitoring of peptidoglycan biosynthesis and high-throughput transpeptidation assays. Nat. Chem. 11, 335-341 (2019).

41. Kuru, E. et al. In situ probing of newly synthesized peptidoglycan in live bacteria with fluorescent D-amino acids. Angew. Chem. Int. Ed. 51, 12519-12523 (2012).

42. Kuru, E. et al. Mechanisms of incorporation for D-amino acid probes that target peptidoglycan biosynthesis. ACS Chem. Biol. 14, 2745-2756 (2019).

43. Hsu, Y. P. et al. Full color palette of fluorescent D-amino acids for in situ labeling of bacterial cell walls. Chem. Sci. 8, 6313-6321 (2017).

44. Agnihotri, S. \& Zadeh, G. Metabolic reprogramming in glioblastoma: the influence of cancer metabolism on epigenetics and unanswered questions. Neur. Oncol. 18, 160-172 (2015).

45. Chen, J., Zhang, C., Mi, Y., Chen, F. \& Du, D. CREB1 regulates glucose transport of glioma cell line U87 by targeting GLUT1. Mol. Cell. Biochem. 436, 79-86 (2017).

46. Lin, C. W. et al. Photosensitization, uptake and retention of phenoxazine Nile blue derivatives in human bladder carcinoma cells. Cancer Res. 51, 1109-1116 (1991).

47. $\mathrm{Hu}, \mathrm{F}$. et al. Visualization and in situ ablation of intracellular bacterial pathogens through metabolic labeling. Angew. Chem. Int. Ed. 58, 1-6 (2019).

48. Zhang, S. Q. et al. Near-IR/visible-emitting thiophenyl-based Ru(II) complexes: efficient photodynamic therapy, cellular uptake, and DNA binding. Inorg. Chem. 58, 14244-14259 (2019).

49. Ronnett, G. V., Hester, L. D., Nye, J. S. \& Snyder, S. H. Human cerebral cortical cell lines from patients with unilateral megalencephaly and Rasmussen's encephalitis. Neuroscience 63, 1081-1099 (1994).

50. Stepp, H. \& Stummer, W. 5-ALA in the management of malignant glioma. Lasers Surg. Med. 50, 399-419 (2018).

51. Yang, X. J. et al. A novel zebrafish xenotransplantation model for study of glioma stem cell invasion. PLoS ONE 8, e61801 (2013).
52. Vittori, M., Breznik, B., Hrovat, K., Kenig, S. \& Lah, T. T. RECQ1 helicase silencing decreases the tumour growth rate of U87 glioblastoma cell xenografts in zebrafish embryos. Genes 8, 222-233 (2017).

53. Varga-Patron, L. A. et al. Xenotransplantation of human glioblastoma in zebrafish larvae: in vivo imaging and proliferation assessment. Biol. Open 8 , bio043257 (2019).

54. Chilakamarthi, U. \& Giribabu, L. Photodynamic therapy: past, present and future. Chem. Rec. 17, 775-802 (2017).

55. Ducorthial, G. et al. Development of a real-time flexible multiphoton microendoscope for label-free imaging in a live animal. Sci. Rep. 5, 18303-18315 (2015).

56. Dilipkumar, A. et al. Label-free multiphoton endomicroscopy for minimally invasive in vivo imaging. Adv. Sci. 6, 1801735-1801743 (2019).

57. Akram, A. R. et al. In situ identification of Gram-negative bacteria in human lungs using a topical fluorescence peptide targeting lipid A. Sci. Transl. Med. 10, eaal00333 (2018).

58. Zaar, O., Sjöholm Hylén, A., Gillstedt, M. \& Paoli, J. A prospective, randomized, within subject study of ALA-PDT for actinic keratosis using different irradiation regimes. Photodermatol. Photoimmunol. Photomed. 34, 338-342 (2018).

59. Anderson, C. M. H. et al. Transport of the photodynamic therapy agent 5aminolevulinic acid by distinct $\mathrm{H}+$-coupled nutrient carriers coexpressed in the small intestine. J. Pharmacol. Exp. Ther. 332, 220-228 (2010).

60. Westerfield M. The Zebrafish Book: A Guide for the Laboratory Use of Zebrafish (Danio rerio) 4th edn (Univ. Oregon Press, 2000).

61. Redmond, R. \& Gamblin, J. N. A compilation of singlet oxygen yields from biologically relevant molecules. Photochem. Photobiol. 70, 391-475 (1999)

62. Zhang, Y. H. et al. AIE based GSH activatable photosensitizer for imagingguided photodynamic therapy. Chem. Commun. 56, 10317-10320 (2020).

63. Fernandez, J. M., Bilgin, M. D. \& Grossweiner, L. I. Singlet oxygen generation by photodynamic agents. J. Photochem. Photobiol. B 37, 131-140 (1997).

\section{Acknowledgements}

The authors acknowledge funding from Medical Research Scotland (S.B.: 879-2015), MSCA Individual Fellowship (A.F.: 704912), Wellcome Trust Sir Henry Dale Fellowship (Y.F.: 100104/Z/12/Z), Cancer Research UK Early Detection Award (Y.F.: C38363/ A26931), the Medical Research Council (A.M.E.: MR/N013166/1) and the National Research Foundation by the Ministry of Science, ICT \& Future Planning, South Korea (J-S.L.: NRF-2018M3A9H4079286). M.V. acknowledges funds from ERC Consolidator Grant (771443). The authors thank the technical support from the Flow Cytometry, the Confocal Advanced Light Microscopy, and the BVS aquatic facility units at the University of Edinburgh.

\section{Author contributions}

S.B., F.d.M., and A.F performed chemical synthesis and characterization of the compounds. J-.S.L. carried out DFT calculations. E.K., N.L.A., and J-.S.L. designed and performed bacterial experiments. S.B. designed and performed experiments in eukaryotic cells. L.H. and D.S. provided cell lines. I.R.B., A.M.E., and Y.F. performed zebrafish experiments. S.B. and M.V. wrote the manuscript with contributions from all authors. M.V. supervised the overall project.

\section{Competing interests}

The University of Edinburgh has submitted the patent application "Small molecule photosensitizers for photodynamic therapy" (ID: WO2020/187913) covering some of the work described in this manuscript, where S.B., F.d.M, A.F., and M.V. are named as inventors. The remaining authors declare no competing interests.

\section{Additional information}

Supplementary information The online version contains supplementary material available at https://doi.org/10.1038/s41467-021-22578-2.

Correspondence and requests for materials should be addressed to Y.F. or M.V.

Peer review information Nature Communications thanks Xiaoyuan Chen and the other, anonymous, reviewer(s) for their contribution to the peer review of this work. Peer reviewer reports are available.

Reprints and permission information is available at http://www.nature.com/reprints

Publisher's note Springer Nature remains neutral with regard to jurisdictional claims in published maps and institutional affiliations. 
(c) (i) Open Access This article is licensed under a Creative Commons Attribution 4.0 International License, which permits use, sharing, adaptation, distribution and reproduction in any medium or format, as long as you give appropriate credit to the original author(s) and the source, provide a link to the Creative Commons license, and indicate if changes were made. The images or other third party material in this article are included in the article's Creative Commons license, unless indicated otherwise in a credit line to the material. If material is not included in the article's Creative Commons license and your intended use is not permitted by statutory regulation or exceeds the permitted use, you will need to obtain permission directly from the copyright holder. To view a copy of this license, visit http://creativecommons.org/ licenses/by/4.0/.

(C) The Author(s) 2021 